\title{
Ultrasonic velocity models in liquids (micro- and nanofluids): theoretical validations
}

\author{
S. Nithiyanantham ${ }^{1}$
}

Received: 22 July 2018 / Accepted: 15 March 2019 / Published online: 27 March 2019

(c) The Author(s) 2019

\begin{abstract}
The theoretical ultrasonic velocity in any micro/nano fluids is computed in connection with the literatures. Kudriatsav theory (KT), Jouyban-Acree model (JAM), Floty theory (FT), Ramasamy-Anbanantham model (RAM), Glinkski model (GM), McAllister model (Mc-AM), time average model (TAM), Tavlorides groups (TG), Urick model (UM), Kuster and Toksoz model and modified Urick model (MUM) methods are computed. Further the validity of those theory to identify or check or confirm the possibility of existence of type of interaction, ideal and non-ideal behavior of the system was explained in the basis of hydrogen bonding interaction, induced dipoles interactions. The values have been computed for the pure solute and solvent with the binary, ternary, and pentenary mixtures above the marginal range of miscibility at various temperatures. The models/theory is relevant to the type of fluids and the medium such as ionic, electronic, micelle, aqueous or any type of liquids. The structure breaks the bonds in the associated molecules into their components by means of temperature. The measured parameters are fitted with their polynomial relations to compute the coefficients and standard errors for the validation of the experimental results. McAllister three bodies and multibody models were used to correlate their properties at various temperatures showing association/disassociation nature.
\end{abstract}

Keywords Ultrasonic velocity $\cdot$ Theoretical models $\cdot$ Liquid mixtures $\cdot$ Molecular interactions $\cdot$ Nanofluids

\section{Introduction}

The field of atomic and molecular acoustics relates the macroscopic parameters of sound waves and their properties of the propagating medium. And so, the studies of velocity and adsorption in sound give detail about the properties of gases, liquids such as in much different dispersion. The dependence temperature and concentration dependence of acoustic studies pave some significant observations of intermolecular interaction in liquid medium and solutions, since the liquid mixtures were found applications in the area of medical, technology, agriculture, fertilizers, restorations of food and related industries [1, 2]. In recent times, the ultrasonic analysis found wide applications in determining the physicomechanical (solids), physicochemical and bio-physical behavior

S. Nithiyanantham

s_nithu59@rediffmail.com

1 Post Graduate \& Research Department of Physics (Ultrasonics/NDT Divisions), Thiru. Vi. Kalyanasundaram Govt Arts and Science College (Affiliated to Bharathidasan University, Tiruchirappalli), Thiruvarur 610003, India of variety of liquid mixtures [3, 4]. The acoustical with thermodynamic properties evaluated in the in liquids mixtures, which are not obtained by any other methods which may be useful mainly in automobiles like combustion, petrol and diesel. Further, confirming the existence of specific interaction, the authors try to relate the values from experimental findings with those predicted molecular models and to know the molecular insight. This comparison gives fruitful information about the mixtures to test the validity of many empirical theories for the mixtures of binary, ternary [5, 6], tertiary and sometimes pentenary [4]. The thermodynamic dependence of fluids is more useful for sustaining the properties (density, viscosity) at high temperatures with more mechanical operations worldwide like train engine working continuously more than $100 \mathrm{~h}$. The race car and high-speed motors run many revolutions per minute (tribology). It creates more temperature, absorbing heat from components of mixtures and remove the heat frequently by nanofluids. In recent time's nano-lubricants, nano-coolant plays a vital role for high-speed mechanical operations.

The cohesive and adhesive properties of liquids indicate the nature of suspended solid particles and also indicate the 
intermediate properties of gas and solids. So, the liquid possesses the intermediate physical properties of solid and gasses, collectively called as condensed matter. The idea behind the liquid is considered as disordered solid and as condensed gas and so the pair potential parameter is accountable [7, 8].

Based on the molecular structure, Eyring proposed that the idea of condensed matter behavior and Hilderbrand commented and refused to consider a solid [9]. The molecular movement sometimes favors a condensed state, but the thermal energy agitates and destroys the actual structure. The concluding analysis is based on the molecular theories like perturbation, spherical particle, etc. [10]. And so, the acceptable partition function cannot be obtained due to many structures of particles other than sphere.

The nature of the molecules can be studied in liquids and liquid mixtures with statistical analysis from the standard references. Besides the available models, some new models can be proposed. However, the aim is to investigate the interaction, catalytic activity, molecular-kinetic nature for the assumed systems; it is much important to select and apply one model among those existing models. If the available models are not suitable to validate the experimental results, we can propose new model suitable for experiment with valid assumption. Evaluating ultrasonic velocity values computed from various models in liquid mixtures are compared and validate with suitable following model [11]. These models are Kudriatsav theory (KT), Jouyban-Acree model (JAM), Floty theory (FT), Ramasamy-Anbanantham model (RAM), Glinkski model (GM), Mc. Alister model (Mc-AM), time average model (TAM), Tavlorides groups (TG), Urick model (UM), Kuster and Toksoz model and modified Urick model (MUM). Also, the percentage deviation, standard percentage error and Chi-squared test are computed [12-31].

While making the solution and for calculation of the percentage composition of the constituent of liquid mixtures [32-34], to find the strength of molecular interactions of liquid mixtures, through the non-rectilinear behavior of ultrasonic velocity. To obtain the additional information about the nature and strength of interaction of molecule, other parameters related to ultrasonic velocity can be used. The important features of ultrasonic velocity in experiment and the relevant experimental model are non-invariant, precision, rapidly and easy automation. Several researchers reported on ultrasonic velocity with models used for computing ultrasonic velocity [12-31].

\section{Experimental}

The Analar Grade chemicals are purified with normal procedures $[32,33]$. And proper mixtures are prepared with mole fractions and the experimental ultrasonic velocities are measured with ultrasonic interferometer of any made, with an accuracy of $\Delta U \pm 10^{-2} \mathrm{~ms}^{-1}$. The density of liquid mixtures was determined with densitometer and the viscosity of the samples was measured with Ostwald's viscometer with the accuracy of $\Delta \rho= \pm 10^{-6} \mathrm{~cm}^{-3}$ and $\Delta \eta= \pm 0.001 \mathrm{~kg} \mathrm{~m}^{-3}$, respectively [34]. Further, it is completely free from air and bubbles (complete solutions). The particle sizes can be determined with laser diffractions/any other conventional methods suitable to the nature of solutions.

\section{Theories/models}

\section{Kudriavtsev theory $\left(U_{K T}\right)$}

Kudriavtsev theory [12] gives the velocity theoretically $U_{\mathrm{KT}}$, through the following relation:

$U_{\mathrm{KT}}^{2}=X_{\mathrm{A}}\left(\frac{M_{\mathrm{A}}}{\bar{M}}\right) U_{\mathrm{A}}^{2}+X_{\mathrm{B}}\left(\frac{M_{\mathrm{B}}}{\bar{M}}\right) U_{\mathrm{B}}^{2}+5.5 \times 10^{8} L$,

where $M_{\mathrm{A}}, M_{\mathrm{B}}$, and $M$ represent the mol. wts. of components $A$ and $B$, the effective molecular weight $M_{\text {eff }}=\left(X_{\mathrm{A}} M_{\mathrm{A}}+X_{\mathrm{B}} M_{\mathrm{B}}\right), L$ represents the heat capacity of the mixture in $\mathrm{cal} / \mathrm{g}$.

\section{Jouyban-Acree model $\left(U_{J A}\right)$}

Jouyban-Acree and his groups $[13,14]$ recently correlated viscosity; density is further extended to velocity by the units of Mehdi Hasan [15]. According to his assumption, the velocity due to JA model is given by $j$ :

$$
\begin{aligned}
\ln U_{\mathrm{JA}}= & X_{1} \ln U_{1}+X_{2} \ln U_{2}+\left(X_{1} X_{2} / T\right) \\
& \times\left\{A_{0}+A_{1}\left(X_{1}-X_{2}\right)+A_{1}\left(X_{1}-X_{2}\right)^{2}\right\} .
\end{aligned}
$$

Here, $U_{1}$ and $U_{2}$ are velocities of pure liquids 1 and 2. $X_{1}$ and $X_{2}$ are the mole fractions of 1 and 2 correspondingly.

\section{Flory's theory $\left(U_{\mathrm{FT}}\right)$}

A significant method from surface tension of mixtures is the Flory based on statistics [16]. Patterson and Rastogi proposed the model with reduced parameters [17]. The values of ultrasonic velocity from surface tension, temperature, pressure, volume with their reduced parameters through Auerbeck relation [35]

To calculate the characteristic surface tension [5],

$\sigma *=K^{1 / 3} P *^{2 / 3} T *^{1 / 3}$,

where, $K, P^{*}$ and $T^{*}$ are the Boltzmann constant, pressure and temperature of corresponding characteristics nature of fluids. Here

$P *=\frac{\alpha T V^{2}}{k_{\mathrm{T}}}$, 
where ' $\alpha$ ' is the thermal expansion coefficient and ' $k_{\mathrm{T}}$ ' is the isothermal compressibility,

$\alpha=\frac{75.6 \times 10^{-3}}{T \frac{1}{9} u_{\mathrm{m}}^{\frac{1}{2}} \rho_{m}^{\frac{1}{2}}}$,

$$
k_{\mathrm{T}}=\frac{1.71 \times 10^{-3}}{T^{\frac{4}{9}} \rho_{\mathrm{m}}^{\frac{4}{3}} u_{\mathrm{m}}^{2}} .
$$

The reduced volume $V$ through ' $\alpha$ ' is from the following expression:

$V=\left[1+\left(\frac{\alpha T}{3(1+\alpha T)}\right)\right]^{3}$.

The characteristic temperature $T^{*}$ is given as:

$T *=T\left(\frac{V^{\frac{4}{3}}}{V^{\frac{1}{3}}-1}\right)$.

where the characteristic and reduced parameters are used to compute the surface tension of binary liquid mixtures:

$V_{\mathrm{m}}^{*}=x_{1} V_{1}^{*}+x_{2} V_{2}^{*}$,

$V_{\mathrm{m}}=\frac{V_{\mathrm{m}}}{\left\{x_{1} V_{1}^{*}+x_{2} V_{2}^{*}\right\}}$,

$P *=\psi_{1} P_{1}^{*}+\psi_{2} P_{2}^{*}-\psi_{1} \theta_{2} X_{12}$

$T *=\frac{P *}{\left[\frac{\psi_{1} P_{1}^{*}}{T_{1}^{*}}+\frac{\psi_{2} P_{2}^{*}}{T_{2}^{*}}\right]}$.

where $\psi$ is the segment fraction, $\theta_{2}$ is the site fraction, and $X_{12}$ is the interaction parameters:

$$
\begin{aligned}
& \psi_{1}=\frac{x_{1} V_{1}^{*}}{x_{1} V_{1}^{*}+x_{2} V_{2}^{*}}, \\
& \psi_{2}=1-\psi_{1}, \\
& \theta_{2}=\frac{\psi_{2}}{\left[\psi_{2}+\psi_{1}\left(\frac{V_{2}^{*}}{V_{1}^{*}}\right)^{1 / 3}\right]}, \\
& X_{12}=P_{1}^{*}\left[1-\left(\frac{V_{2}^{*}}{V_{1}^{*}}\right)^{\frac{1}{6}}\left(\frac{P_{2}^{*}}{P_{1}^{*}}\right)^{\frac{1}{2}}\right]^{2} .
\end{aligned}
$$

The 'reduced surface tension' is given by Priogogonie and Saraga [36]:

$\sigma(V)=M V^{-\frac{5}{3}}-\frac{V^{\frac{1}{3}}-1}{V^{2}} \ln \left[\frac{V^{\frac{1}{3}}-0.5}{V^{\frac{1}{3}}-1}\right]$,

where $M$ is the fraction of nearest neighbors, the molecule moving from the bulk to the surface. And the surface tension $(\sigma)$ of a mixture is

$\sigma_{\mathrm{m}}=\sigma * \sigma(V)$.

The surface tension from Flory theory has been used to evaluate ultrasonic velocity of mixture, with the help of familiar Auerback relation [16, 35]:

$u_{\mathrm{m}}=\left(\frac{\sigma_{\mathrm{m}}}{6.3 \times 10^{-4} \rho_{\mathrm{m}}}\right)^{2 / 3}$.

\section{Ramasamy and Anbanantham model ( $\left.U_{\text {RAM }}\right)$}

Ramasamy and Anbananthan [18] proposed the theory based on the idea of impedance parameter with the mole fraction of components. With equilibrium condition:

$A+B \leftrightarrow A B$

and the constant of association $K_{\text {as }}$ is

$K_{\mathrm{as}}=\frac{[A B]}{[A][B]}$,

where $[A]$ is the amount of solvent and $[B]$ is the amount of solute in the liquid mixture.

By applying the linearity condition,

$U_{\mathrm{obs}}=X_{\mathrm{A}} U_{\mathrm{A}}+X_{\mathrm{AB}} U_{\mathrm{AB}}$,

where $X_{\mathrm{A}}, X_{\mathrm{AB}}, U_{\mathrm{A}}$ and $U_{\mathrm{AB}}$ are the mole fraction of component $A$, and associative $A B$, ultrasonic velocity of component $A$ and associate $A B$ and observed ultrasonic velocity, respectively, and sometimes with non-equilibrium condition. With the existence of non-associated component, the above expression becomes:

$U_{\mathrm{RA}}=\left[X_{\mathrm{A}} U_{\mathrm{A}}+X_{\mathrm{B}} U_{\mathrm{B}}+X_{\mathrm{AB}} U_{\mathrm{AB}}\right]$,

where $X_{\mathrm{B}}$ and $U_{\mathrm{B}}$ are the mole fractions of component $B$ and ultrasonic velocity of $B$ (non-associated component).

$K_{\text {as }}=\frac{[A B]}{\left(C_{\mathrm{A}}-[A B]\right)\left(C_{\mathrm{B}}-[A B]\right)}$,

where $C_{\mathrm{A}}$ and $C_{\mathrm{B}}$ are initial molar concentrations of the components. The value of $K_{\text {as }}$ computes the equilibrium value of $[A B]$ for every composition of the mixture as well as $[a]=\mathrm{C}_{\mathrm{A}}-[A B]$ and $[B]=C B-[A B]$.

$$
K_{\mathrm{as}}=\frac{a_{\mathrm{AB}}}{\left(a_{\mathrm{A}}-a_{\mathrm{AB}}\right)\left(a_{\mathrm{B}}-a_{\mathrm{AB}}\right)}
$$


where $a_{\mathrm{A}}, a_{\mathrm{B}}$ and $a_{\mathrm{AB}}$ are the additivity of components $A$, $B$ and associate $A B$ with equimolar which may be equal to

$a_{\mathrm{A}}^{\prime}=a_{\mathrm{A}}-a_{\mathrm{AB}}$ and $a_{B}^{\prime}=a_{\mathrm{B}}-a_{\mathrm{AB}}$,

where $a_{\mathrm{A}}^{\prime}$ and $a_{\mathrm{B}}^{\prime}$ are the activities of $[A]$ and $[B]$ in equimolar quantities.

From Eq. (25), $K_{\mathrm{as}}$ is

$$
K_{\mathrm{as}}=\frac{a_{\mathrm{AB}}}{a_{\mathrm{A}} a_{\mathrm{B}}-a_{\mathrm{A}} a_{\mathrm{AB}}-a_{\mathrm{B}} a_{\mathrm{AB}}+a_{\mathrm{AB}}^{2}}=\frac{a_{\mathrm{AB}}}{a_{\mathrm{A}}^{\prime} \cdot a_{\mathrm{B}}^{\prime}} .
$$

At now, consider if any value of ultrasonic velocity in the hypothetical pure component $A B, U_{\mathrm{AB}}$, it is possible to equate the ultrasonic velocity calculated using Eq. (26) with the experiment.

$S=\sum\left(U_{\mathrm{obs}}-U_{\mathrm{cal}}\right)^{2}$

where $U_{\text {obs }}$ and $U_{\text {cal }}$ are the observed and calculated equilibrium properties, respectively.

The low values of $S$ can be obtained theoretically by a pair of fitted parameters. But, we found that for some $K_{\text {as }}$ and $U_{\mathrm{AB}}$, the values of $S$ are high and varied rapidly, and for others, it is low and changes occur slowly when changing the fitted parameters. In these types, the value of $U_{\mathrm{AB}}$ is not much lower/higher than the lowest/highest observed ultrasonic velocity of the system. Quantitatively, it should be accepted with reasonable parameters $K_{\mathrm{as}}$ and $U_{\mathrm{AB}}$ which has the physical meaning and which reproduces satisfactorily experimental properties.

\section{Glinkski model $\left(U_{G M}\right)$}

On analyzing the ultrasonic velocity values from Ramaswamy and Anbananthan model, Glinski [19] suggested the additive function with the volume fraction, $\phi$ of the components, the extracted form by Natta and Baccaredda model [20] as:

$U_{\mathrm{GL}}=\frac{U_{\mathrm{A}} U_{\mathrm{B}} U_{\mathrm{AB}}}{\phi_{\mathrm{A}} U_{\mathrm{A}} U_{\mathrm{AB}}+\phi_{\mathrm{B}} U_{\mathrm{A}} U_{\mathrm{A}} B_{\phi}+\phi_{\mathrm{AB}} U_{\mathrm{A}} U_{\mathrm{B}}}$,

where $U_{\mathrm{GL}}$ is the theoretical ultrasonic velocity of binary liquid mixtures, $\phi_{\mathrm{A}}$ and $\phi_{\mathrm{B}}$ are the volume fractions of components $A$ and $B$ and $U_{\mathrm{A}}, U_{\mathrm{B}}$ and $U_{\mathrm{AB}}$ are the ultrasonic velocity of components $A, B$ and $A B$. The numerical procedure, computation and calculation of constant are same as described before. In addition to this, model which was already mentioned was further developed and elaborated by many researchers $[21,22,37]$.

\section{McAllister 3-body model $\left(U_{\text {McA-3 }}\right)$}

McAllister assumed in the study of the viscosity of a mixture of molecules type (1) and (2). And he proposed that the total free energy of activation will be dependent on $\left(\Delta G_{i i}, \Delta G_{i j}\right.$ or $\left.\Delta S_{i j k}\right)$ of individual interactions, with their fraction and the total occurrences $\left(x_{i}^{3}, x_{i}^{2} x_{j}, x_{j}^{2}, x_{i} x_{j}^{2}\right.$ or $\left.x_{i} x_{j} x_{k}\right)$. Hence

$$
\begin{aligned}
\Delta G *= & x_{1}^{2} \Delta G_{1}^{*}+x_{1}^{2} x_{2} \Delta G_{121}^{*}+2 x_{1}^{2} x_{2} \Delta G_{112}^{*} \\
& +x_{1} x_{2}^{2} \Delta G_{212}^{*}+2 x_{1} x_{2}^{2} \Delta G_{123}^{*}+x_{2}^{3} \Delta G_{2}^{*} .
\end{aligned}
$$

In another way

$\Delta G^{*}=x_{1}^{3} \Delta G_{1}^{*}+3 x_{1}^{2} x_{2} \Delta G_{12}^{*}+3 x_{1} x_{2}^{2} \Delta G_{12}^{*}+x_{2} \Delta G_{2}^{*}$.

Now, applying Eq. (30) for each set of possible interactions (i.e., 111, 121, .., and 222) and then taking logarithms of equations obtained and removing free energy terms,

$$
\begin{aligned}
\ln u= & x_{1}^{3} \ln u_{1}+3 x_{1}^{2} x_{2} \ln u_{12}+3 x_{1} x_{2} \ln u_{21} \\
& +x_{2}^{3} \ln u_{2}-\ln \left[x_{1}+x_{2} M_{2} / M_{1}\right] \\
& +3 x_{1}^{2} x_{2} \ln \left[\left(2+M_{2} / M_{1}\right) / 3\right] \\
& +3 x_{1} x_{2}^{2} \ln \left[\left(1+2 M_{2} / M_{1}\right) / 3\right] \\
& +x_{2}^{3} \ln \left[M_{2} / M_{1}\right]
\end{aligned}
$$

where,

$M_{12}=\frac{2 M_{1}+M_{2}}{3}$,

and

$M_{21}=\frac{2 M_{2}+M_{2}}{3}$.

\section{McAllister 4-body model $\left(U_{\text {MCA-4 }}\right)$}

If the molecular size is somewhat more differed, the 4 body system seems to be 3 body systems. Further, considering different interactions, the occurrence of free energy is the sum of all activations energy.

$$
\begin{aligned}
\Delta G *= & x_{1}^{4} \Delta G_{1}^{*}+4 x_{1}^{3} \times 2 \Delta G_{2221}^{*} \\
& +6 x_{1}^{2} x_{2}^{2} \Delta G_{2221}^{*} 4 x_{1} x_{2}^{3} \Delta G_{2221}^{*}+x_{2}^{3} \Delta G_{2}^{*} .
\end{aligned}
$$

By the techniques entirely similar to method in (34), it becomes

$$
\begin{array}{r}
\ln u_{\text {mix }}=x_{1}^{4} \ln u_{1}+4 x_{1}^{3} x_{2} \Delta \ln u_{1112}+6 x_{1}^{2} x_{2} \ln u_{1122}+4 x_{1} x_{2}^{2} \ln u_{222}+x_{2}^{4} \ln u_{2} \\
-\ln \left(x_{1}+x_{2} M_{2} / M_{1}\right)+4 x_{1}^{3} x_{2} \ln \left[\left(3+M_{2} / M_{1}\right) / 4\right]+6 x_{1}^{2} x_{2}^{2} \ln \left[\left(1+M_{2} / M_{1}\right) / 2\right] \\
+4 x_{1} x_{2}^{3} \ln \left[\left(1+3 M_{2} / M_{1}\right) / 4\right]+x_{2}^{4} \ln \left(M_{2} / M_{1}\right),
\end{array}
$$


where all the notations having the usual meaning.

\section{Time average model $\left(\boldsymbol{U}_{\mathrm{tAvg}}\right)$}

In time average model, the reflection with refraction effects is neglected.

$\phi=\left(t *-t_{\mathrm{c}}\right) /\left(t_{\mathrm{d}}-t_{\mathrm{c}}\right)$.

\section{Tavlarides and co-workers model $\left(U_{\mathrm{TM}}\right)$}

Tavlarides and their co-workers proposed this model, and here the effects of reflection and refraction phenomenon are accountable. The coefficients $g_{\mathrm{d}}$ and $g_{\mathrm{c}}$ depend on the ratio $\gamma$ and subsequently the model produces branches depending on phase continuity [23, 24],

$\phi=\frac{t *-t_{\mathrm{c}}}{g_{\mathrm{d}} t_{\mathrm{d}}-g_{\mathrm{c}} t_{\mathrm{c}}}$,

$\gamma=\frac{\text { speed of sound in dispersed space }}{\text { speed of sound in continous space }}$,

for $\gamma \geq 1$

$g_{\mathrm{c}}=1+\frac{1}{r^{3}}\left[1-\left(1-\gamma^{2}\right)^{3 / 2}\right]-\frac{3}{5 \gamma^{3}}\left[1-\left(1-\gamma^{2}\right)^{5 / 2}\right]-\frac{2}{5} \gamma^{2}$,

$g_{\mathrm{d}}=\frac{1}{\gamma^{2}}\left[1-\left(1-\gamma^{2}\right)^{3 / 2}\right]$,

for $\gamma \leq 1$

$$
\begin{aligned}
g_{c}= & 1+\frac{2}{5 \gamma^{3}}-\gamma^{2}\left[1-\left(1-\frac{1}{\gamma^{2}}\right)^{3 / 2}\right] \\
& +\frac{3}{5} \gamma^{2}\left[1-\left(\frac{1}{\gamma^{2}}\right)^{5 / 2}\right]-\left(1-\frac{1}{\gamma^{2}}\right)^{3 / 2},
\end{aligned}
$$

$g_{\mathrm{d}}=\frac{1}{\gamma^{2}}$.

\section{Urick model $\left(U_{U r}\right)$}

In this Urick model, the particles are assumed to be very small compared to the sound wavelength and so the effect of scattering and the velocity of sound can be omitted [25]. Density and compressibility values are found averages weighted by the phase concentration:

$u=\frac{1}{\sqrt{\rho * k_{\mathrm{m}}}}$, $\frac{\rho_{\mathrm{c}}-\rho *}{\rho_{\mathrm{c}}+2 \rho *}=\phi \frac{\rho_{\mathrm{c}}-\rho_{\mathrm{d}}}{\rho_{\mathrm{c}}+2 \rho_{\mathrm{d}}}$,

$k_{\mathrm{m}}=\phi k_{\mathrm{d}}+(1-\phi) k_{\mathrm{c}}$.

\section{Kuster and Toksoz model ( $\left.U_{\mathrm{KUT}}\right)$}

In this model, the dispersed solid with spherical and the phase is non-viscous fluid. With the same above assumption of particle size with wavelength of sound, multiple scattering effects are negligible and so it gives two branches [26]:

$U=\frac{1}{\sqrt{\rho_{\mathrm{m}} * k_{\mathrm{m}}}}$,

$\frac{\rho_{\mathrm{c}}-\rho *}{\rho_{\mathrm{c}}+2 \rho *}=\phi \frac{\rho_{\mathrm{c}}-\rho_{\mathrm{d}}}{\rho_{c}+2 \rho_{\mathrm{d}}}$

$k_{\mathrm{m}}=\phi k_{\mathrm{d}}+(1-\phi) k_{\mathrm{c}}$.

\section{Modified Urick model $\left(U_{\text {MUr }}\right)$}

In this model, they account the scattering by thermal means, but applying the long wavelength limit with the two branches from phase continuity [27-30].

From Urick equation, $u=\frac{1}{\sqrt{\rho_{\mathrm{m}} k_{\mathrm{m}}}}$, after substituting $p_{\mathrm{m}}$ and $k_{\mathrm{m}}$, wee obtain

$u=\frac{1}{\sqrt{\left(\phi \rho_{\mathrm{d}}+(1-\phi) \rho_{\mathrm{c}}\right)\left(\phi k_{\mathrm{d}}+(1-\phi) k_{\mathrm{c}}\right.}}$.

In general, the speed of sound in the continuous phase is

$u_{\mathrm{c}}=\frac{1}{\sqrt{\rho_{\mathrm{c}} k_{\mathrm{c}}}}$.

Squaring and dividing Eqs. (50) by (49), and making a series, algebraic transformation is

$$
\begin{aligned}
\frac{u_{\mathrm{c}}^{2}}{u^{2}} & =\frac{\left.\left(\phi \rho_{\mathrm{d}}+1-\phi\right) \rho_{\mathrm{c}}\right)\left(\phi k_{\mathrm{d}}+(1-\phi) k_{\mathrm{c}}\right)}{\rho_{\mathrm{c}} k_{\mathrm{c}}} \\
& =\frac{\left.\left(\left(\rho_{\mathrm{d}}-\rho_{\mathrm{c}}\right) \phi \rho_{\mathrm{c}}\right)\left(k_{\mathrm{d}}-k_{\mathrm{c}}\right) \phi+k_{c}\right)}{\rho_{\mathrm{c}} k_{\mathrm{c}}} \\
& =\frac{\rho_{\mathrm{c}} k_{\mathrm{c}}+\left(k_{\mathrm{c}}\left(\rho_{\mathrm{d}}-\rho_{\mathrm{c}}\right)+\rho_{\mathrm{c}}\left(k_{\mathrm{d}}-k_{\mathrm{c}}\right)\right) \phi+\left(\rho_{\mathrm{d}}-\rho_{c}\right)\left(k_{\mathrm{d}}-k_{\mathrm{c}}\right) \phi_{2}}{\rho_{\mathrm{c}} k_{\mathrm{c}}} \\
& =1+\left(\frac{\rho_{\mathrm{d}}-\rho_{\mathrm{c}}}{\rho_{\mathrm{c}}}+\frac{k_{\mathrm{d}}-k_{\mathrm{c}}}{k_{\mathrm{c}}}\right) \phi+\left(\frac{\rho_{\mathrm{d}}-\rho_{\mathrm{c}}}{\rho_{\mathrm{c}}}\right)\left(\frac{k_{\mathrm{d}}-k_{\mathrm{c}}}{k_{\mathrm{c}}}\right) \phi^{2} .
\end{aligned}
$$


Hence,

$$
\begin{aligned}
\frac{1}{u^{2}}= & \frac{1}{u_{\mathrm{c}}^{2}}\left(1+\left(\frac{k_{\mathrm{d}}-k_{\mathrm{c}}}{k_{\mathrm{c}}}+\frac{\rho_{\mathrm{d}}-\rho_{\mathrm{c}}}{\rho_{\mathrm{c}}}\right) \phi\right. \\
& \left.+\left(\frac{k_{\mathrm{d}}-k_{\mathrm{c}}}{k_{\mathrm{c}}}\right)\left(\frac{\rho_{\mathrm{d}}-\rho_{\mathrm{c}}}{\rho_{\mathrm{c}}}\right) \phi^{2}\right) .
\end{aligned}
$$

Or in simple (introducing $\alpha_{1}$ and $\delta_{1}$ as coefficients in front of $\phi$ and $\phi^{2}$ terms);

$\frac{1}{u_{2}}=\frac{1}{u_{c}^{2}}\left(1+\alpha_{1} \phi+\delta_{1} \phi^{2}\right)$.

The other form of modified Urick Eq. (55) is

$\frac{1}{u_{2}}=\frac{1}{u_{c}^{2}}\left(1+\alpha \phi+\delta \phi^{2}\right)$

$\alpha=\left(\frac{k_{\mathrm{d}}-k_{\mathrm{c}}}{k_{\mathrm{c}}}+\theta+\frac{\rho_{\mathrm{d}}-\rho_{\mathrm{c}}}{\rho_{\mathrm{c}}}\right)$

$\delta=\left(\frac{k_{\mathrm{d}}-k_{\mathrm{c}}}{k_{\mathrm{c}}}+\theta\right)\left(\frac{\rho_{\mathrm{d}}-\rho_{\mathrm{c}}}{\rho_{\mathrm{c}}}\right)+\frac{2\left(\rho_{\mathrm{d}}-\rho_{\mathrm{c}}\right)^{2}}{3 \rho_{\mathrm{c}}^{2}}$

$\theta=(\mu-1) \frac{\rho_{\mathrm{d}} C_{\mathrm{pd}}}{\rho_{\mathrm{c}} C_{\mathrm{pc}}} R^{2}$ and $R=\left(\frac{\beta_{\mathrm{d}}}{\rho_{\mathrm{d}} C_{\mathrm{pd}}}-\frac{\beta_{\mathrm{c}}}{\rho_{\mathrm{c}} C_{p c}}\right) / \frac{\beta_{\mathrm{c}}}{\rho_{\mathrm{c}} C_{\mathrm{pc}}}$.

\section{Validations}

Among the all above models, the validations of some models were illustrated as follows, the time-average model, which equivalent to the sound propagation through a the medium is considered as layer by layer, (ii) In this modified timeaverage model by Taylaries and co-workers [23, 24], (iii) Urick model [25], (iv) model of Kuster and Toksoz [26] and (v) modified Urick model [27, 28].

Meng and his co-workers studied the above models for water with oil at temperatures $25^{\circ} \mathrm{C}, 40^{\circ} \mathrm{C}$ and $55^{\circ} \mathrm{C}$ [29]. For the comparison of models (i)-(iv), model (i) based on the time-average approach is not a sufficient model to describe the behavior of oil-water mixtures studied here. Because in this model there is no consideration of any scattering effects, it is slightly concentrated with high dispersion media (droplets). The models (iii) and (iv) seem to match the experimental data most closely. The phase inversions of oil and water occur at the content values of 30-70\% and that inversion causes due to the thermodynamic conditions. The application of model $(\mathrm{v})$ is considering scattering by thermal energy of thermal with $\beta$, specific heat capacities, $C_{\mathrm{p}}$ and $C_{\mathrm{v}}$, for both continuous and non-continuous medium.

The effect of specific heat capacities for hydrocarbons of petrochemicals like crude oil and water in this experiments reports is generally a mixture of many hydrocarbons and the properties are similar $[30,31,38,39]$. From the model (v), the view of $\theta$ depends on the magnitude of factor $(\mu-1)$, mostly which is small in case of fluids for water and very large molecules of hydrocarbons. The effect of temperature and the changes in $C_{\mathrm{p}}, \mu_{\text {water }}=1, \mu_{\text {oil }}=1$ become $\mu_{\text {water }}>1$ and $\mu_{\text {oil }}>0$.

The Ramasamy and Anbanantham model [18] was corrected by Glinski [19] and tested by Natta and Baccaredda [40] to predict direct and the related associational behavior of liquid mixtures. The quantities analyzed were refractive index, molar volume, viscosity, free length, free volume, internal pressure, compressibility and many [35-37, 40, 41]. The results are fitted with the adjustable parameters. The values of $K_{\mathrm{as}}, U A$ and $B$ are the fitted parameters. On changing the parameters, the equilibrium concentrations of individual components $[A],[B]$ and $[A B]$ change and the ultrasonic velocity can be computed. The difference in experiment and theory values for ultrasonic velocity is used to obtain the sum of squares of deviation. The values of ultrasonic velocity in pure associate can be treated as a fitted one with the values of $K_{\mathrm{as}}$.

Values of $(\alpha)$ and $K_{\mathrm{s}}$ are needed in the PFP model obtained from the equations which have been tested already in many cases [36, 37, 41-43]. The advantage of the Flory theory over others is that the essential parameters required in this theory can be precisely determined using the physical parameters of pure liquids [44]; the average percentage deviations of the ultrasonic velocity obtained by the Flory statistical theory for both the mixtures have been discussed [45]. The ultrasonic velocities of both the mixtures were also analyzed in terms of the Flory statistical theory using Eq. (19). The APD of the comparison of ultrasonic velocity are reported in et al. [46] and it is found good agreement between the experimental and calculated values of $u$ with a minimum APD of -0.81 for the THF $+1-\mathrm{P}$ mixture and a maximum APD of -5.15 for the THF + 2-P mixture [46]. It may be concluded that the statistical mechanical theory can be used as a powerful tool to evaluate the excess functions directly from the ultrasonic velocity and density data.

This is common the deviations in any parameter (ultrasonic velocity) can be mentioned by Redlich-Kishter polynomial equation [47] for correlating the experimental data as:

$y=x_{i}\left(1-x_{i}\right) \sum_{i=o}^{p} A_{i}\left(2 x_{i}-1\right)^{i}$

where ' $y$ ' shows deviation in ultrasonic velocity, $x_{i}$ is the mole fraction and $A_{i}$ is the coefficient of $i$ th component, respectively. The coefficient values can be computed through multiple regression analysis (least square) and are summarized along with the standard deviations.

The reduced Redlich-Kister excess properties are expressed as: 
$Q_{y . \text { exp }},\left(x_{1}\right)=Y^{E} /\left(x_{1}\left(1-x_{1}\right)\right)$,

where $Y^{E}$ denotes any excess values (excess ultrasonic velocity). The reduced Redlich-Kister ( $R R-K)$ functions give much better insight information of the non-ideality. i.e., RRK excess property is more sensitive than direct excess property occurring at low concentrations [48, 49]. The liquid mixtures having specific interactions like association at low concentrations with different size of molecules (clusters). And in the RRK- polynomials remove the effect of excess functions and gives specific reduced functions in (55) characterizing the velocity and other property and also gives evidence to the existence of important interactions [48, 49].

Combined nearly ideal binary solvent/Redlich-kister equation $(\mathrm{CNIBS} / \mathrm{R}-\mathrm{K})$ is

$\ln X_{\mathrm{m}}=f c \ln X c+f w \ln X w+f c f w \Sigma w(f c-f w)$.

The solubility behavior of liquid mixtures with organic solvent is from $i=0$ to $i=3$ [50]. And it is also able to describe the multiple solubility maximum and solubility at various temperatures [51]. The miscibility and solubility are the key factors while designing the drugs in mixed solvents [52].

McAllister coefficient $a, b$ and $c$ were calculated using the least square procedure. With the variation in mole fractions, the values of speed of sound obtained from all the models decrease in trend at all temperatures.

The observations leading to the associated components parameters provide better agreement over the non-associated components' parameters. It means that larger deviation values in PFP model can be explained as the model was developed for non-electrolyte $\gamma$-meric spherical chain molecules and the system under investigation has interacting and associating properties, Even though the formula used for this computation of $\alpha$ and $\beta_{\mathrm{T}}$ is also empirical in general. The molecular function and complex functions lead to positive deviations in speed of sound, whereas negative deviations are due to molecular dissociation. The sign and magnitude of deviations in sound depend on the relative strength of the two opposite effects. The less smoothness in deviations is due to the interaction between the existing component molecules. Isentropic compressibility shows increasing trend with the increase in mole fraction, and the density and ultrasonic velocity show other behavior [46].

The recently developed Juoyban-Acree model is applied successfully to the mixture of ortho, meta, para cresols and 1,4 dioxane, aniline and pyridine; these correlations in all six systems further fitted with Redlich-Kister Polynomials. From the analysis, Juoyban-Acree model is fitted better over than other studied models [53]. The spectroscopic analysis such as FTIR and UV-Visible absorption study can support to confirm the acoustical results obtained from various derived and theoretical parameters. And it is applicable to some alcohols and electrolytes [54-57].

\section{Conclusions}

Here, the author taken twelve no of theory/model of ultrasonic velocities for various liquid mixtures and are validated with experimental observations. The trends in observation of any one model suggest highly suitable with particular mixtures, either ideal, non-ideal and strength with magnitude of interactions of confirmed. Overall, further it is stated that all the models used in this present investigation successfully agree well with the experimental values and findings, and its shows that the liquids should have poor associating properties. In future, a general equation is fit to explain the liquid property more reliable and acceptable for associate and nonassociated parameters of many fluids at nano and all ranges.

Open Access This article is distributed under the terms of the Creative Commons Attribution 4.0 International License (http://creativeco mmons.org/licenses/by/4.0/), which permits unrestricted use, distribution, and reproduction in any medium, provided you give appropriate credit to the original author(s) and the source, provide a link to the Creative Commons license, and indicate if changes were made.

\section{References}

1. Tamura, K., Sanada, T., Murakani, S.: Thermodynamic properties of aqueous solution of 2-isopropoxyethanol at $25^{\circ} \mathrm{C}$. J. Solut. Chem. 28, 777 (1999)

2. Gargia, B., Alcalde, R., Lea, J.M., Mahs, J.S.: Shear viscosities of the $N$-methylformamide- and $N, N$-dimethylformamide- $\left(\mathrm{C}_{1}-\mathrm{C}_{10}\right)$ alkan1-ol solvent systems. J Chem Soc Faradays Trans. 93, 1115 (1999)

3. Sarvazya, A.P.: Ultrasonic velocimetry of biological compounds. Annu. Rev. Biophys. Biophys. Chem. 20, 321-342 (1991)

4. Oswal, S.L., Oswal, P., Phalak, P.D.: Speed of sound, isentropic compressibilities, and excess molar volumes of binary mixtures containing $p$-dioxane. J. Solut. Chem. 27, 507 (1998)

5. Jorg, M., Ghoneim, A., Turky, G., Stockhausen, M.: Dielectric relaxation of some $N, N$-disubstituted amides. Phys Chem Liqs 29, 263 (1995)

6. Nithiyanantham, S., Palaniappan, L.: Thermodynamic studies of lactose with amylase in aqueous media at 298.15 K. J Comput Theor NanoSci 9, 2190-2192 (2012)

7. Nithiyanantham, S.: Structural and bio-molecular interaction studies of starch $+\alpha$-amylase at 298.15 K. J Comput Theor NanoSci 12, 2048-2061 (2015)

8. Henry, M.: Nonempirical quantification of molecular interactions in supramolecular assemblies. Chem. Phys. Chem. 3, 561-569 (2002)

9. Hilderbrand, J.H.: Motions of molecules in liquids: viscosity and diffusivity. Science 174, 490-493 (1971)

10. Nithiyanantham, S.: Ultrasonic velocity models in liquids (nanofluids). J. Comput. Theor. Nanosci. 14(5), 2077-2082 (2017)

11. Van Deal, W., Vangeal, E.: Proceeding of the First International Conference on Calorimetry and thermodynamics, Warsaw, p. 556 (1969)

12. Kudriavtsev, B.B.: Sov. Phys. Acoust. 14, 298 (1956)

13. Jouybon, A., Kboubhsabjafari, M., Gharamalaki, Z.V., Fekari, Z., Acree, W.E.: Calculation of the viscosity of binary liquids at various temperatures using Jouyban-Acree model. J. Chem. Pharma. Bull. 53, 53 (2005)

14. Juoyban, A., Aazabayzani, A.F., Acree, W.E.: Surface tension calculation of mixed solvents with respect to solvent composition and 
temperature by using Jouyban-Acree model. J. Chem. Pharma. Bull. 52, 1219 (2004)

15. Hasan, M., Shirude, D.F., Hiray, A.P., Sawant, A.B., Kadam, U.B.: Densities, viscosities and ultrasonic velocities of binary mixtures of methylbenzene with hexan-2-ol, heptan-2-ol and octan-2-ol at $T=298.15$ and 308.15 K. Fluid Phase Equlib. 252, 88 (2007)

16. Flory, P.J.: Statistical thermodynamics of liquid mixtures. J. Am. Chem. Soc. 87, 1883 (1965)

17. Patterson, D., Rastogi, A.K.: The surface tension of polyatomic liquids and the principle of corresponding states. J. Phys. Chem. 74, 1067 (1970)

18. Ramasamy, K., Anbananthan, D.: Acustica. 48, 281-282 (1981)

19. Glinski, J.: Determination of the conditional association constants from the sound velocity data in binary liquid mixtures. J. Chem. Phys. 118, 2301-2307 (2003)

20. Aralaguppi, M.I., Jadar, C., Aminabhavi, T.M.: Density, viscosity, refractive index, and speed of sound in binary mixtures of 2-chloroethanol with methyl acetate, ethyl acetate, $n$-propyl acetate, and $n$-butyl acetate. J. Chem. Eng. Data 44, 441 (1999)

21. Shukla, R.K., Awasthi, N., Kumar, A., Shukla, A., Pandey, V.K.: Prediction of associational behaviour of binary liquid mixtures from viscosity data at $298.15,303.15,308.15$ and $313.15 \mathrm{~K}$. J. Mol. Liq. 158, 131 (2011)

22. Ali, A., Tariq, M.: Surface thermodynamic behaviour of binary liquid mixtures of benzene + 1,1,2,2-tetrachloroethane at different temperatures: an experimental and theoretical study. Phys. Chem. Liq. 46, 47 (2008)

23. Tsouris, C., Talvarides, L.L.: Volume fraction measurements of water in oil by an ultrasonic technique. Ind. Eng. Chem. Res. 32, 998-1002 (1993)

24. Tsouris, C., Norato, M.A., Tavlarides, L.L.: A pulse-echo ultrasonic probe for local volume fraction measurements in liquidliquid dispersions. Ind. Eng. Chem. Res. 34, 3154-3158 (1995)

25. Urick, R.J.: A sound velocity method for determining the compressibility of finely divided substances. J. Appl. Phys. 18, 983987 (1947)

26. Kuster, G.T., Toskoz, M.N.: Velocity and attenuation of seismic waves in two-phase media: part I. theoretical formulations. Geophysics 39, 587-606 (1974)

27. Pinfield, V.J., Povey, M.J.W.: Thermal scattering must be accounted for in the determination of adiabatic compressibility. J. Phys. Chem. B. 101, 1110-1112 (1997)

28. Pinfield, V.J., Povey, M.J.W., Dickinson, E.: The application of modified forms of the Urick equation to the interpretation of ultrasound velocity in scattering systems. Ultrasonics 3, 243-251 (1995)

29. Meng, G., Jaworski, A.J., White, N.M.: Composition measurements of crude oil and process water emulsions using thick-film ultrasonic transducers. Chem. Eng. Process. 45, 383-391 (2006)

30. US National Institute of Standards and Technology.: NIST Chemistry Web Book-NIST Standard Reference Database Number 69. http://webbook.nist.gov/chemistry/fluid/ (2003)

31. Dovnar, D.V., Lebedinskij, Y.A., Khasanshin, T.S., Shcemelev, A.P.: The thermodynamic properties of n-pentadecane in the liquid state, determined by the results of measurements of sound velocity. High Temp. 39(6), 835-839 (2001)

32. Rao, K.P., Reddy, K.J.: Excess volumes and excess isentropic compressibilities of binary mixtures of $\mathrm{N}, \mathrm{N}$ - dimethylformamide with branched alcohols at $303.15 \mathrm{k}$. Thermochim. Acta 91, 321 (1985)

33. Weissberger, A., Pronahar, F.S., Riddick, J.A., Toops, E.E.: Texts of Organic Chemistry, Vol. II Organic Solvents. Inderscience, New York (1955)

34. Riddick, J.A., Bunger, W.B., Sakano, T.: Organic Solvents; Physical Properties and Methods of Purification, 4th edn. Wiley Inderscience, New York (1986)
35. Auerbach, N.: Surface tension and speed of sound. Experientia 4, 473 (1948)

36. Prigogine, I., Saraga, L.: On the surface tension and the cell model of liquid states. J. Chem. Phys. 49, 399-407 (1952)

37. Reis, J.C.R., Santos, A.F.S., Disas, F.A., Lampreia, I.M.S.: Correlated volume fluctuations in binary liquid mixtures from isothermal compressions at 298.15 K. Chem. Phys. Chem. 9, 1178-1188 (2008)

38. Khasanshin, T.S., Shchemelev, A.P.: The thermodynamic properties of n-tetradecane in liquid state. High Temp. 40(2), 207-211 (2002)

39. Khasanshin, T.S., Sehamialion, A.P., Poddubskij, O.G.: Thermodynamic properties of heavy $n$-alkanes in the liquid state: $n$-dodecane. Int. J. Thermophys. 24(5), 1277-1289 (2003)

40. Natta, G., Baccaredda, M.: Sulla velocita di propagazione degli ultrasuoni nelle miscele ideali. Atti Accad. Naz. Lincei 4, 360 (1948)

41. Glasstone, S., Laidler, K., Eyring, H.: The Theory of Rate Process. McGraw-Hill, New York (1941)

42. Shukla, R.K., Kumar, A., Srivastava, K., Singh, N.: A comparative study of the PFP and BAB models in predicting the surface and transport properties of liquid ternary systems. J. Solut. Chem. 36, 1103-1116 (2007)

43. Pandey, J.D., Chandra, P., Srivastava, T., Soni, N.K., Singh, A.K.: Estimation of surface tension of ternary liquid systems by corresponding-states group-contributions method and Flory theory. Fluid Phase Equlib. 273, 44-51 (2008)

44. Gupta, M., Vibhu, I., Shukla, J.P.: Ultrasonic velocity, viscosity and excess properties of binary mixture of tetrahydrofuran with 1-propanol and 2-propanol. Fluid Phase Equilib. 244, 26 (2006)

45. Singh, S., Vibhu, I., Gupta, M., Shukla, J.P.: Excess acoustical and volumetric properties and the theoretical estimation of the excess thermodynamic functions of binary liquid mixtures. Chin. J. Phys. 5(4), 412-424 (2007)

46. Kumar, A., Srivastava, U., Singh, A.K., Srivastava, K., Shukla, R.K.: Sound velocity and isentropic compressibility of binary liquid systems from various theoretical models at temperature range 293.15 to 313.15 K. Can. Chem. Trans. 4(2), 157-167 (2016)

47. Redlich, O., Kister, A.T.: Algebraic representation of thermodynamic properties and the classification of. Ind. Eng. Chem. 40, 345-348 (1948)

48. Desnoyers, J.E., Perron, G.: Treatment of excess thermodynamic quantities for liquid mixtures. J. Solut. Chem. 26(8), 749-755 (1997)

49. Ouerfelli, N., Bouanz, M.: A shear viscosity study of cerium (III) nitrate in concentrated aqueous solutions at different temperatures. J. Phys. Condens. Matter 8, 2763-2774 (1996)

50. Jouyban-Gharamaleki, A., Valaee, L., Barzegar-Jalali, M., Clark, B.J., Acree Jr., W.E.: Comparison of various cosolvency models for calculating solute solubility in water-cosolvent mixtures. Int. J. Pharm. 177, 93-101 (1999)

51. Jouyban-Gharamaleki, A.: The modified Wilson model and predicting drug solubility in water-cosolvent mixtures. Chem. Pharm. Bull. 46, 1058-1061 (1998)

52. Jouyban-Gharamaleki, A., Acree Jr., W.E.: Prediction of drug solubility in ethanol-ethyl acetate mixtures at various temperatures using the Jouyban-Acree model. Int. J. Pharm. 167, 177-182 (1998)

53. Nageswara Rao, J.: Ultrasonic study of molecular interactions in liquid mixtures. M.Phil thesis, S.V. University, Tirupati (2010)

54. Md Nayeem, S., Kondaiah, M., Sreekanth, K., Krishna Rao, D.: Ultrasonic investigations of molecular interaction in binary mixtures of cyclohexanone with isomers of butanol. J. Appl. Chem. 2014, 11 (2014). (Article ID 741795)

55. Punitha, S., Uvarani, R., Pannerselvam, A., Nithiyanantham, S.: Physico-chemical studies on some saccharides in aqueous 
cellulose solutions at different temperatures - acoustical and FTIR analysis. J. Saudi Chem. Soc. 18(5), 657 (2014)

56. Eyring, H., John, M.S.: Significance of liquid structures. Wiley Eastern, New York (1969)

57. Pfeiffer, H., Heremans, K.: The sound velocity in ideal liquid mixtures from thermal volume fluctuations. Chem. Phys. Chem. 6, 697-705 (2005)
Publisher's Note Springer Nature remains neutral with regard to jurisdictional claims in published maps and institutional affiliations. 